\title{
Effect of annealing in the Sb and In distribution of type II GaAsSb-capped InAs quantum dots
}

\author{
D F Reyes , J M Ulloa , A Guzman , A Hierro , D L Sales , R Beanland, \\ A M Sanchez and D González
}

\begin{abstract}
Type II emission optoelectronic devices using GaAsSb strain reduction layers (SRL) over InAs quantum dots (QDs) have aroused great interest. Recent studies have demonstrated an extraordinary increase in photoluminescence (PL) intensity maintaining type II emission after a rapid thermal anneal (RTA), but with an undesirable blueshift. In this work, we have characterized the effect of RTA on InAs/GaAs QDs embedded in a SRL of GaAsSb by transmission electron microscopy (TEM) and finite element simulations. We find that annealing alters both the distribution of Sb in the SRL as well as the exchange of cations (In and $\mathrm{Ga}$ ) between the QDs and the SRL. First, annealing causes modifications in the capping layer, reducing its thickness but maintaining the maximum $\mathrm{Sb}$ content and improving its homogeneity. In addition, the formation of Sb-rich clusters with loop dislocations is noticed, which seems not to be an impediment for an increased PL intensity. Second, RTA produces flatter QDs with larger base diameter and induces a more homogeneous QD height distribution. The Sb is accumulated over the QDs and the RTA enlarges the Sb-rich region, but the Sb contents are very similar. This fact leaves the type II alignment without major changes. Atomic-scale strain analysis of the nanostructures reveal a strong intermixing of $\mathrm{In} / \mathrm{Ga}$ between the QDs and the capping layer, which is the main responsible mechanism of the PL blueshift. The improvement of the crystalline quality of the capping layer together with higher homogeneity QD sizes could be the origin of the enhancement of the PL emission.
\end{abstract}

Keywords: III-V quantum dots, GaAsSb, Sb distribution, Strain state, annealed, TEM

\section{Introduction}

In recent decades, researchers have focused on the design of telecommunication devices with InAs/GaAs quantum dots (QDs) capable of emitting in the $1.55 \mu \mathrm{m}$ maximum transmittance window of optical fibers. Up to now, it has been difficult to achieve this objective in a simple way and researchers are now testing alternative approaches. One possibility is the modification of the strain state and band offsets of QDs by using different types of coating layers, such as strain compensation layers (SCLs) [1] or strain reduction layers (SRL) [2, 3]. Indeed, SRLs extend the infrared emission since a compressively strained layer reduces hydrostatic deformation and increases biaxial strain in an adjacent QD $[4,5]$. Recently, the use of GaAsSb SRLs has become of interest because $\mathrm{Sb}$ also has the advantage of being a good 
surfactant, thereby suppressing the generation of defects and decomposition of the quantum dots (QDs), while allowing the displacement to the infrared wavelength $[6,7]$. Moreover, $\mathrm{GaAsSb}$ layers have an extra degree of freedom in band structure engineering, since the emission type changes from I to II when $\mathrm{Sb}$ content is above $17 \%$ [7]. Due to the spatial separation of electrons and holes, QDs with type II alignment may be good candidates for the fabrication of QDs solar cells and memories $[8-10]$, and they also can be used for the manufacture of lasers because of the possibility of reducing the threshold current density and tuning both the wavelength and the gain [11-14]. However, the spatial separation of electrons and holes of the type II QDs is also responsible for a lower emission efficiency and this requires type II QDs with good features to overcome this drawback. Furthermore, the difficulty of incorporating $\mathrm{Sb}$ on GaAs has been repeatedly reported in the literature [15]. Indeed, the effect of interdiffusion, which affects the relative composition of $\mathrm{In} / \mathrm{Ga}$ and $\mathrm{As} / \mathrm{Sb}$ in and around the QDs, is still debated [16, 17]. An alternative approach to control the Sb distribution is the use of postgrowth thermal annealing that can be used to tailor the band alignment, the wave function overlaps, and hence the recombination dynamics in the InAs/GaAsSb type II QDs [18]. Recently, we have reported about the effect of rapid thermal annealing (RTA) on this kind of sample where large blueshifts and a strong enhancement of emission efficiency were observed while preserving the long radiative lifetimes [19]. However, there was a lack of knowledge about the processes involved during annealing. In this paper, we analyze by advanced transmission electron microscopy (TEM) techniques the structural and compositional effects produced by RTA in GaAsSb-capped InAs/GaAs type II QDs.

\section{Experimental details}

The growth was carried out in a solid source molecular beam epitaxy (MBE) system using Si doped (100) $\mathrm{n}^{+}$GaAs substrates. QDs were grown using the same conditions in both samples: 2.7 monolayers (MLs) of InAs at $450{ }^{\circ} \mathrm{C}$ and $0.04 \mathrm{ML} \mathrm{s}^{-1}$. After $20 \mathrm{~s}$ of growth interruption, $20 \mathrm{ML}$ of $\mathrm{GaAsSb}$ at $470^{\circ} \mathrm{C}$ were deposited, followed by $10 \mathrm{MLs}$ of GaAs grown at the same temperature. The structure was subsequently covered by $250 \mathrm{~nm}$ of GaAs grown at $580^{\circ} \mathrm{C}$. The nominal $\mathrm{Sb}$ content in the GaAsSb capping layer was $28 \%$. The RTA at $850^{\circ} \mathrm{C}$ during $30 \mathrm{~s}$ was performed under nitrogen atmosphere using a MILA-3000 oven. Hereafter, the as-grown and annealed samples were named Sb28 and Sb28RTA, respectively.

Structural and compositional analyses in cross-section specimens prepared using convectional techniques were performed by TEM. Convectional TEM (CTEM) images were obtained in a JEOL $2000 \mathrm{EX}$ at $200 \mathrm{kV}$. High resolution TEM (HRTEM) for strain state analyses were acquired on a JEOL $2011 \mathrm{LaB}_{6}$ operated at $200 \mathrm{kV}$. Additionally, high resolution high angle annular dark field (HR-HAADF) studies were performed using a doubly aberration corrected TEM/STEM JEOL ARM200F operated at $200 \mathrm{kV}$ and equipped with a

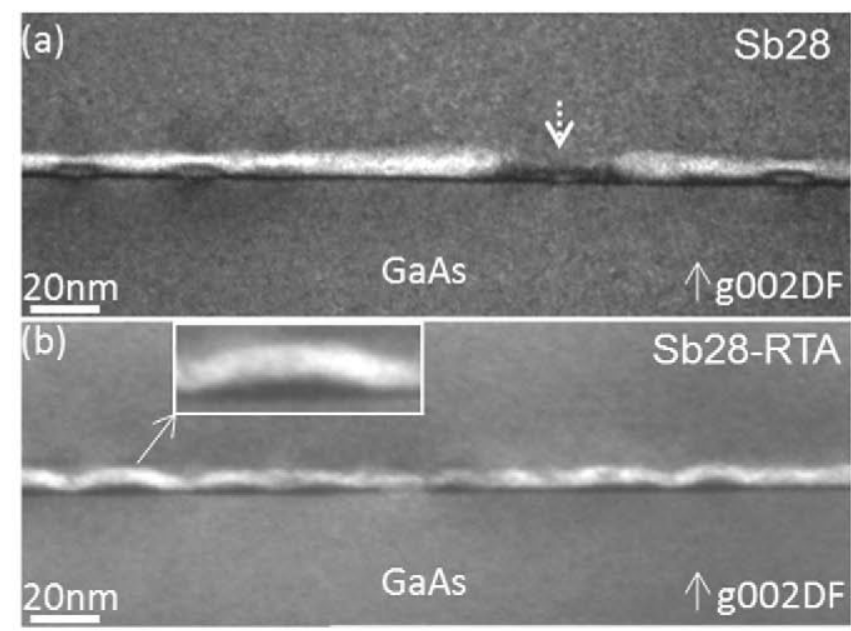

Figure 1. Images $002 \mathrm{DF}$ (a), (b) acquired at [110] pole axis of samples Sb28 and Sb28-RTA, respectively. The QDs and WL display a darker contrast than the GaAs and GaAsSb layer. The inset zoom image of a QD showing how the capping layer is conformed to the QD shape. The arrow marks a discontinuity in the layer, evidenced on a QD in the $\mathbf{S b 2 8}$ sample.

Schottky field emission gun and two CEOS CESCOR hexapole aberration correctors that enable imaging resolution in STEM modes well below $80 \mathrm{pm}$.

\section{Results}

Previous results had shown that the incorporation of a high $\mathrm{Sb}$ content in the SRL extended the emission wavelength of InAs QDs to $1345 \mathrm{~nm}$ (at $15 \mathrm{~K}$ ) and induced a type II band alignment, as revealed by the large radiative lifetime of 14.2 ns (further details of PL and time-resolved PL of these samples can be found in [19]). More important, the RTA retained the type II emission (radiative lifetime of $11.8 \mathrm{~ns}$ ) while producing a significant enhancement of the PL, increasing both the integrated intensity (by a factor of 3.3) and monochromaticity (FWHM reduced from $97 \mathrm{meV}$ to $32 \mathrm{meV}$, i.e., by $67 \%$ ). However, the RTA strongly affected the peak wavelength, producing a blueshift of $220 \mathrm{~nm}$. In order to elucidate the possible reasons for this behavior, the samples were analyzed by TEM techniques to determine the morphological changes induced by RTA, the strain fields, and compositional distribution.

\section{CTEM analyses}

It is well known that QD composition, size, and shape strongly influence the emission wavelength [20-22]. Thus, for example, a reduction in the QD height could explain a blueshift. Hence, compositionally sensitive g200 dark field images were taken of cross-section specimens to analyze the morphological changes produced by the RTA (see figure 1). In these images, the wetting layer (WL) and QDs can be clearly distinguished with a darker contrast than GaAs, while 

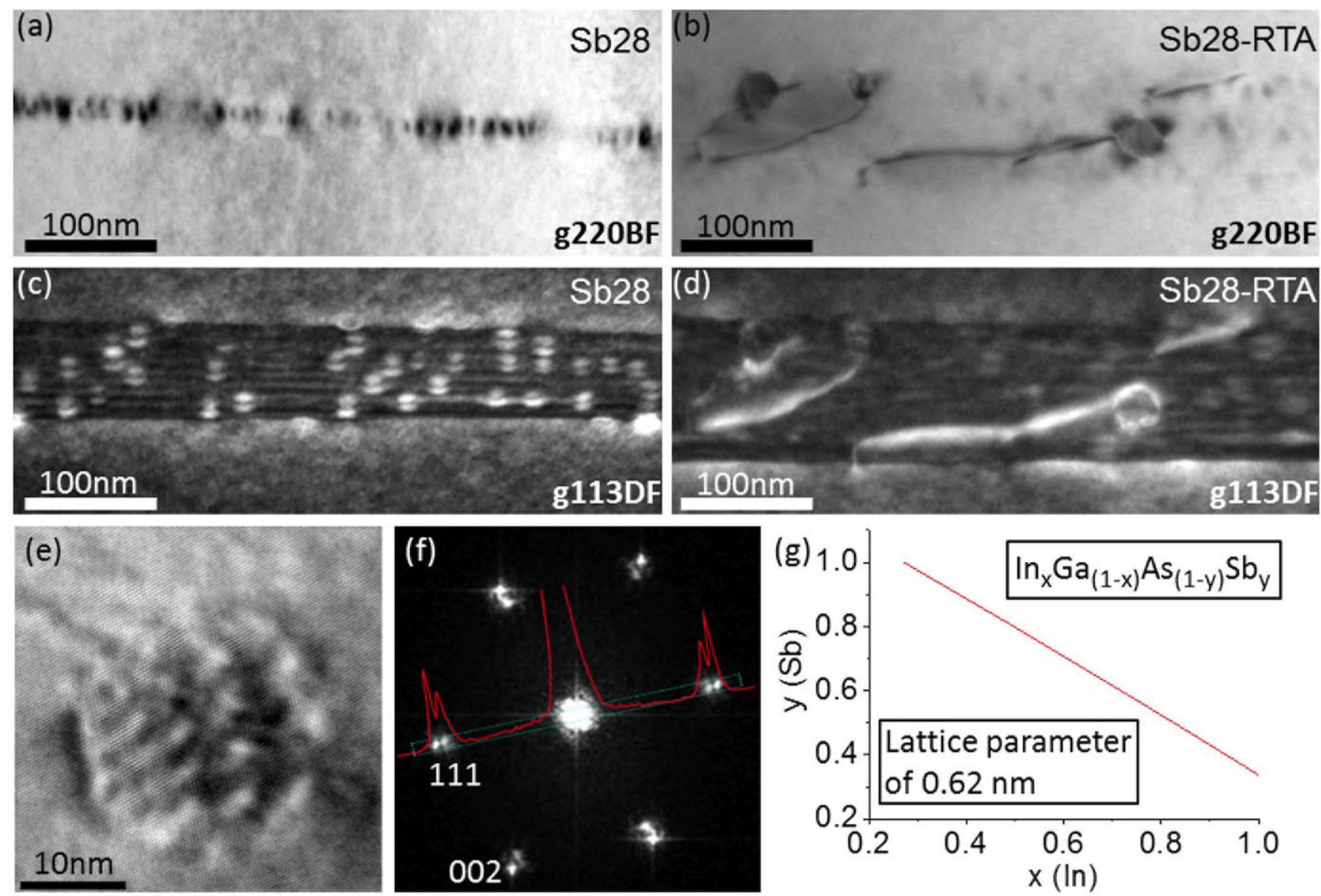

Figure 2. Contrast images acquired after excited diffraction reflections (220) (a), (b), (113) (c), (d) of samples Sb28 and Sb28-RTA. (e), (f) HRTEM images of one cluster and the corresponding fast Fourier transform, respectively, showing the typical zinc-blende pattern of the GaAs matrix and an aligned cluster. $(\mathrm{g})$ Range of $\mathrm{Sb}$ and $\mathrm{In}$ content for its lattice parameter $(0.62 \mathrm{~nm})$.

the capping layer exhibits a brighter contrast. Our measurement on more than 60 QDs [19] showed that the RTA gave way to an important reduction of the average height (from $3.3 \pm 0.6$ to $2.3 \pm 0.4 \mathrm{~nm}$ ) together to an increase of the base diameter (from $16 \pm 4 \mathrm{~nm}$ to $22 \pm 7 \mathrm{~nm}$ ). In addition, the thickness of the SRL was reduced by approximately $1 \mathrm{~nm}$, i.e., from 6.5 to $5.5 \mathrm{~nm}$ in areas far from the QDs. Therefore, the QD aspect ratio decreased to half from 0.206 to 0.104 and the volume increased $30 \%$ in average, with QDs adopting a disk shape. Changes in the morphology of the SRL were also observed. After RTA, the SRL shows undulations in the upper interface adjusting to the shape of the QDs (figure 1(b)). In addition, a decrease of the inhomogeneities over the QDs is observed: the SRL is not able to cover some of the QDs in the as-grown sample (see the arrow in figure 1(a)) but it covers all the QDs homogeneously after RTA.

The RTA produces other structural modifications, in particular the introduction of defects. Figure 2 shows CTEM images of both samples using g220 and g113 reflections. Sample Sb28 shows the typical QD contrast and no extended defects (defect density below $10^{8} \mathrm{~cm}^{-2}$ ). However, for the annealed sample, rounded particles with a strain contrast, consistent with locally different composition, were also observed. These compositional clusters (diameter $15 \pm 4 \mathrm{~nm}$ ) are associated in many cases with dislocation loops being located between regions free of defects (figure 2(b)). The formation of clusters accompanied by dislocation loops after an annealing process has been observed previously in samples of GaAs doped with $\mathrm{Sb}$ and in InAsBi structures [23, 24], where the heaviest element is segregated into clusters and extended defects spread in the growth plane. In our case, the clusters have a zinc-blende structure that is crystallographically aligned to the GaAs matrix as can be seen in the HRTEM image and its fast Fourier transform (figures 2(e) and (f), respectively). Some of them have large lattice parameters up to $0.62 \mathrm{~nm}$ that imply a proportion of In and $\mathrm{Sb}$ as indicated in figure $2(\mathrm{~g})$. These clusters, which must have possibly formed by the aggregation of $\mathrm{Sb}$ and In from the CL and WL, respectively, have a lattice mismatch with the matrix up to $10 \%$ that would explain the formation of the dislocation loops for strain relief. Surprisingly, the formation of Sb-rich clusters in conjunction with dislocation loops does not impair the PL intensity (actually, the peak intensity increases 10 times [19]).

The modifications in size and shape of both QDs and capping layer, as well as the formation of clusters, are the result of diffusion and segregation processes during the annealing. Since the optical properties appear to be unaffected by the clusters and loop dislocations, we focus the detailed 

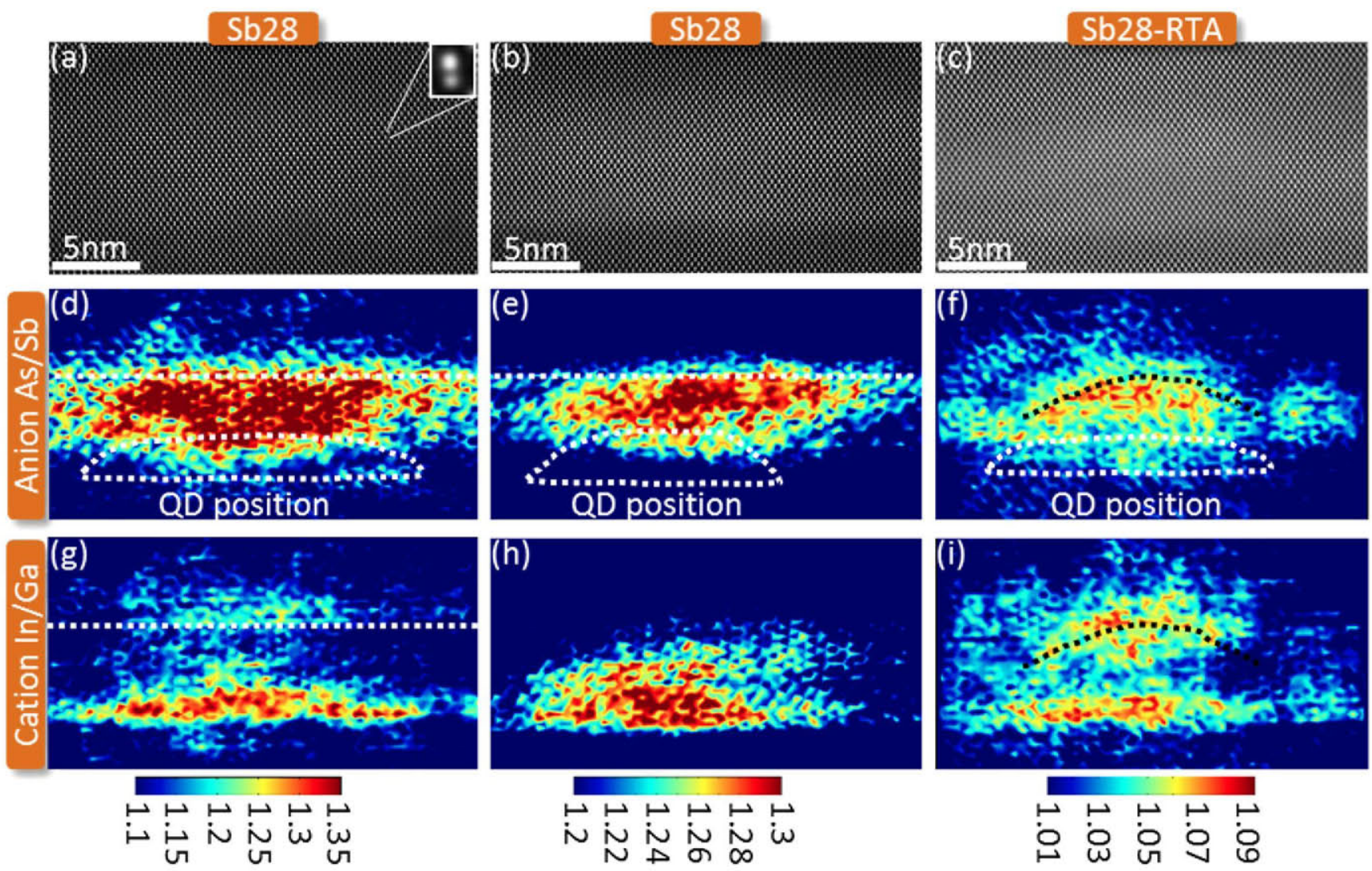

Figure 3. HR-HAADF images acquired in zone axis [110] of the QDs of Sb28 (a), (b) and Sb28-RTA (c) samples and their respective maps of integrated intensities R, anion (d), (e), (f) and cations columns (g), (h), (i). Higher values (hotter colors) are associated with areas with a higher content of heavier elements, In and Sb, respectively, while less intense regions (cooler colors) would correspond to areas of GaAs. The inset in (a) shows a resolved dumbbell and the dashed lines display the position of QDs and the top of the capping layer.

analysis on the morphological and compositional changes of the QDs and SRL.

\section{HR-HAADF analyses}

Although the intensity in $\mathbf{g} 200$ dark field images gives information on composition, it can be difficult to interpret it in the vicinity of the QDs due to its dependence on TEM specimen thickness and strain fields [25, 26]. In addition, as four elements are present simultaneously ( $\mathrm{Ga}$, As, In, and $\mathrm{Sb}$ ), an analysis using only this information is ambiguous [27]. Conversely, in HR-HAADF images such as those shown in the top row of figure 3 , where anions and cations are resolved separately (see inset in figure 3(a)), the distribution of $\mathrm{Sb}$ and In can be assessed independently. The inset shows the resolved cation and anion columns. The second and third rows show maps of normalized anion and cation intensity $[28,29]$. Hotter colors (red) are associated with areas with a higher content of $\mathrm{Sb}$ (figures 4(d)-(f)) or In (figures 4(g)-(i)), while cooler colors (blue) correspond to areas richer in As (figures 4(d)-(f)) or Ga (figures 4(g)-(i)). From left to right, the first two columns refer to the $\mathbf{S b 2 8}$ sample while the third corresponds to the Sb28-RTA sample. Thus, figures 3(d) and (e) show a Sb accumulation over the QD in Sb28. However, two different scenarios are found regarding the In distribution: the more usual situation, where In is confined in the QDs; and a second, where there is a small accumulation of In over the QDs.

The last column of figure 4 reveals the changes of the elemental distribution in the QD and its vicinity produced by annealing. In the annealed sample Sb28-RTA, Sb is present as a lobe over the QDs, but contrary to the $\mathbf{S b 2 8}$ sample, the top of the layer is not flat but undulated. The cation distribution shows an In accumulation over the QDs, with In even above the Sb-containing layer (figures 3(i) and (f)). These results confirm a strong In-Ga intermixing between the QD and the SRL, as well as Sb segregation over the QD. These images of normalized intensities cannot be used to quantify composition, since static atomic displacements associated with strain fields play a major role in HR-HAADF image contrast [30,31]. However, the information provides a qualitative measure of the location of different atoms in the nanostructure.

\section{Strain mapping analysis}

Deformation/strain maps are an additional tool to display the $\mathrm{In} / \mathrm{Sb}$ distribution. HRTEM images were acquired at the $\langle 110\rangle$ zone axis and the strain distribution was obtained by a geometrical phase analysis (GPA) [32]. Figure 4 shows two 

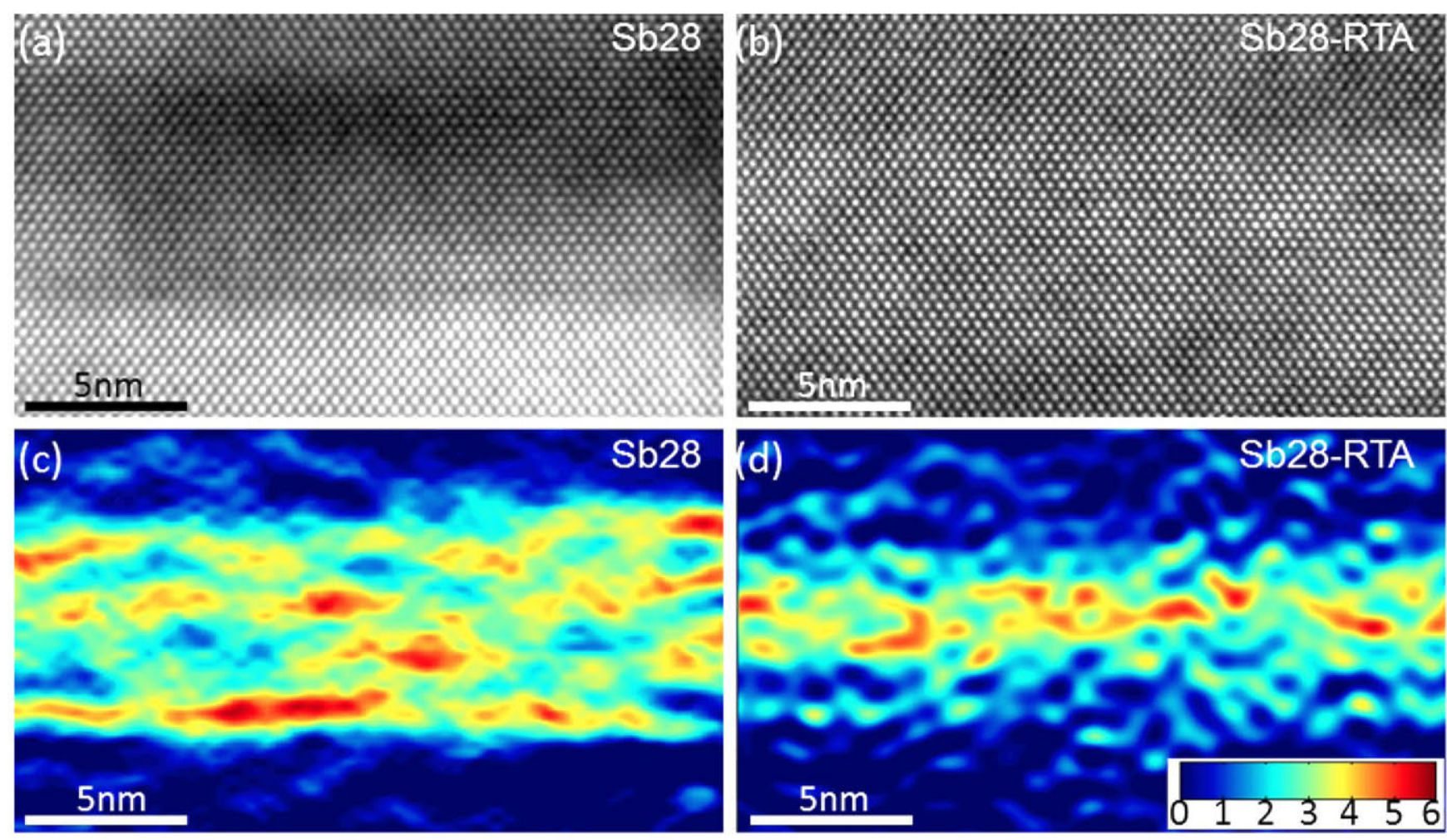

Figure 4. HRTEM images of the capping layer of samples Sb28 (a) and Sb28-RTA (b) in areas far from the QD acquired in the zone axis [110] and their corresponding deformation maps (c), (d). Color scale to $\varepsilon_{\mathrm{zz}}$ deformation expressed in percent.

images of the SRL away from QDs and the corresponding maps of strain along the growth direction $\left(\varepsilon_{z z}\right)$. Indeed, a more strained region due to the surface segregation of $\mathrm{Sb}$ is clearly observed on top of the capping layer [17, 33]. However, the RTA affects all the element distributions in the structure. First, there is a substantial reduction of the average strain $\varepsilon_{z z}$ in the WL, from $(2.5 \pm 0.3) \%$ in $\mathbf{S b 2 8}$ to $(1.6 \pm 0.6) \%$ in $\mathbf{S b 2 8}-$ RTA. The WL is quite blurred, as can be seen in the values of standard deviation of the measurements. Second, the average strains $\varepsilon_{\mathrm{zz}}$ obtained in the upper region of the SRL were very similar, being $3.0 \pm 0.3 \%$ and $2.9 \pm 0.2 \%$ for $\mathbf{S b 2 8}$ and $\mathbf{S b 2 8}$ RTA, respectively. However, the area of low strain between the WL and the GaAsSb layer is thicker after RTA; namely, the Sb-rich region of the SRL is thinner. Remarkably, this fact brings about a considerable reduction of the dispersion of the strain data leading to a more homogeneous distribution of the $\mathrm{Sb}$.

Moreover, the tensile strain $\varepsilon_{z Z}$ in pseudomorphic samples under biaxial stress is due to the tetragonal distortion and it could be used to determine the $\mathrm{Sb}$ content since the GaAs and $\mathrm{GaSb}$ have the same biaxial strain coefficient $\left(R_{\mathrm{B}}=0.899\right)$. Indeed, the average $\mathrm{Sb}$ content in the SRL was estimated assuming the validity of Vegard's law at these contents by using the same procedure described in [29]. Taking all in account, these results imply a similar peak $\mathrm{Sb}$ content in both samples, about $(20 \pm 1) \%$, that is quite far from the nominal value of $28 \%$. Remarkably, there is a substantial reduction of the $\mathrm{Sb}$ in the SRL in the annealed sample, since the layer thickness is shorter. The RTA induces a reduction of the global strain in the region among QDs, by a loss of Sb and In in the SRL and in the WL, respectively.

Figure 5 shows the GPA strain analysis in a region containing a QD in both samples. The RTA affects both the morphology and structure of the QDs and modifies the strain state. A reduction in QD height and the appearance of a region of low strain between the QD and the GaAsSb layer is evident. Additionally, the averaged deformation, $\varepsilon_{z z}$, inside the QDs is very different, $(5.0 \pm 0.2) \%$, for sample Sb28 and significantly lower, $(2.9 \pm 0.4) \%$, in Sb28-RTA, with a reduction of $42 \%$. The reduction of strain $\left(\varepsilon_{z z}\right)$ in the QDs is consistent with the process of minimizing the deformation produced during the RTA. On the other hand, the strain in the SRL over the QDs increases after RTA from 3.3\% in $\mathbf{S b 2 8}$ to $3.9 \%$ in Sb28-RTA. This could be consistent with the In and $\mathrm{Sb}$ distributions observed in the HR-HAADF images. In a first approximation, neglecting the compression effect due to the stress from the QD, the sum of $\mathrm{Sb}$ and In contents over the QDs deduced would be approximately 22 and $26 \%$ in samples Sb28 and Sb28-RTA, respectively. Remarkably, the strain on top of the QD in sample Sb28-RTA is larger than inside the QD, indicating that the RTA process has inverted the QD-CL strain distribution through intermixing. This results in a reduction of the overall strain in the QD-CL region from 8.3 to $6.8 \%$ on average.

All these observations indicate that the RTA induces both In and $\mathrm{Sb}$ segregation. This produces: (i) loss of $\mathrm{Sb}$ and In in the SRL and the WL, respectively, in areas between QDs that give rise to the cluster formation; and (ii) QD decomposition 

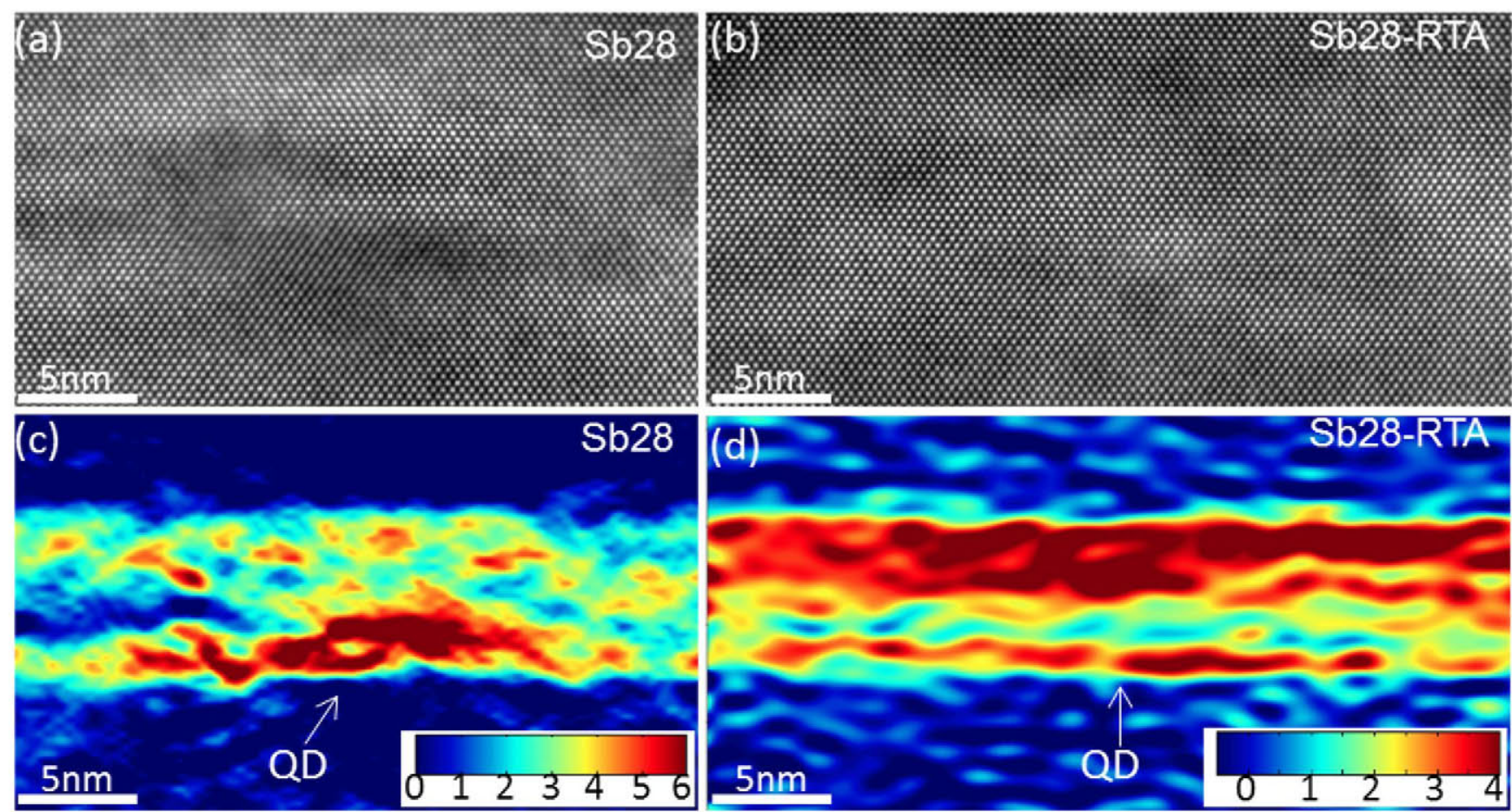

Figure 5. HRTEM images of the QDs of samples Sb28 (a) and Sb28-RTA (b) in areas containing a QD acquired in the zone axis [110] and their corresponding deformation maps (c), (d). Color scale to $\varepsilon_{z z}$ deformation expressed in percent.



Figure 6. Model of the simulated structures of the sample Sb28 (left) and Sb28-RTA (right). A QD can be seen in the center and a lobe containing $\mathrm{Sb}$ and In is placed over the QD in sample Sb28-RTA. The color scheme marks the different domains.

by incorporation of Ga into, with an upper lobe formation due to In and $\mathrm{Sb}$ accumulation.

\section{Discussion}

HRTEM image analysis using GPA provides valuable information about the deformation, and allows us to estimate the composition using elasticity theory. This estimation is more precise away from the QDs, since the morphology is simpler. To understand the strain around the QDs, a theoretical study of the deformation in the system was carried out using finite element methods (FEM) with Comsol Multiphysics ${ }^{\circledR}$ and Matlab® software. Two models using the experimental structures obtained in samples Sb28 and Sb28-RTA were created (figure 6). We also inserted a lobe over the QD in the annealed sample. The Sb content was considered constant, and the QDs were not pure InAs [34, 35]. The In content was assumed to change along the growth direction, with maximum In in the area with highest strain in the experimental deformation maps (i.e., $100 \%$ In for $\mathbf{S b 2 8}$ and $80 \%$ In for Sb28-RTA) [34]. A lattice compression, both above and below the QDs, occurs because of tetragonal distortion and would affect any strain measurement and therefore the estimation of $\mathrm{Sb}$ and $\mathrm{In}$ composition. Taking this compression into account, the $\mathrm{Sb}$ and In contents in the SRL over the QDs should be adjusted from 22 to $27 \%$ in sample $\mathbf{S b 2 8}$ and from 26 to $29 \%$ in sample Sb28-RTA, reducing the difference 

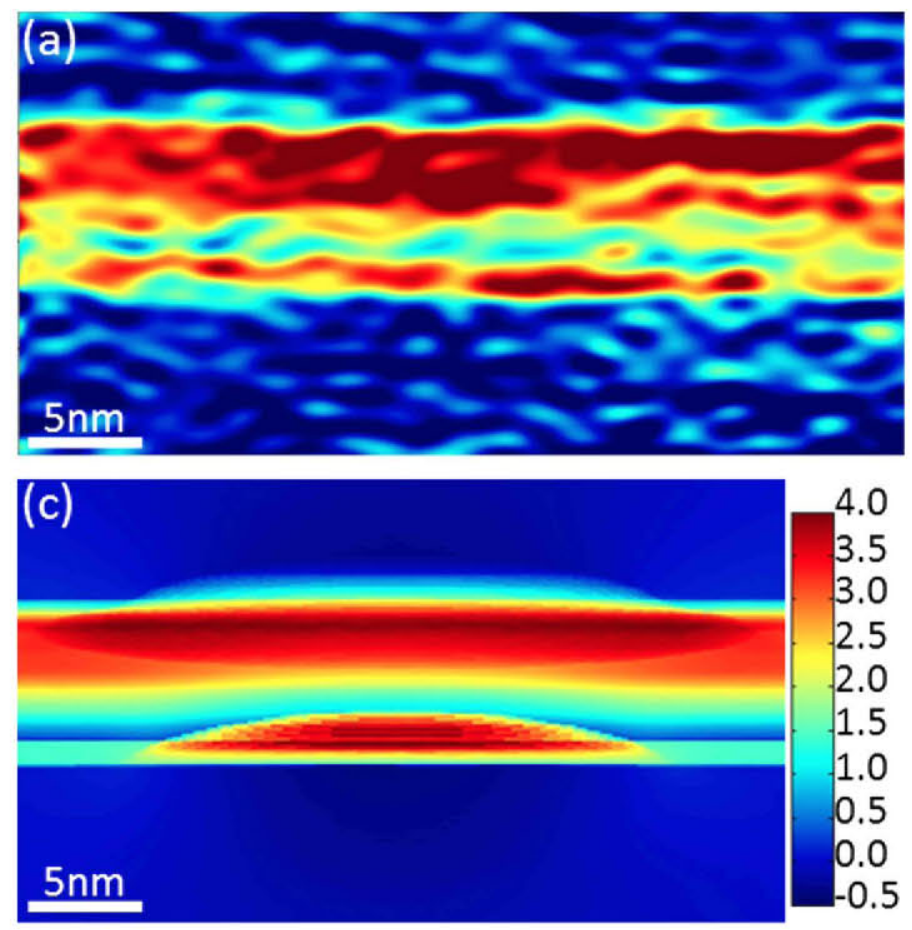
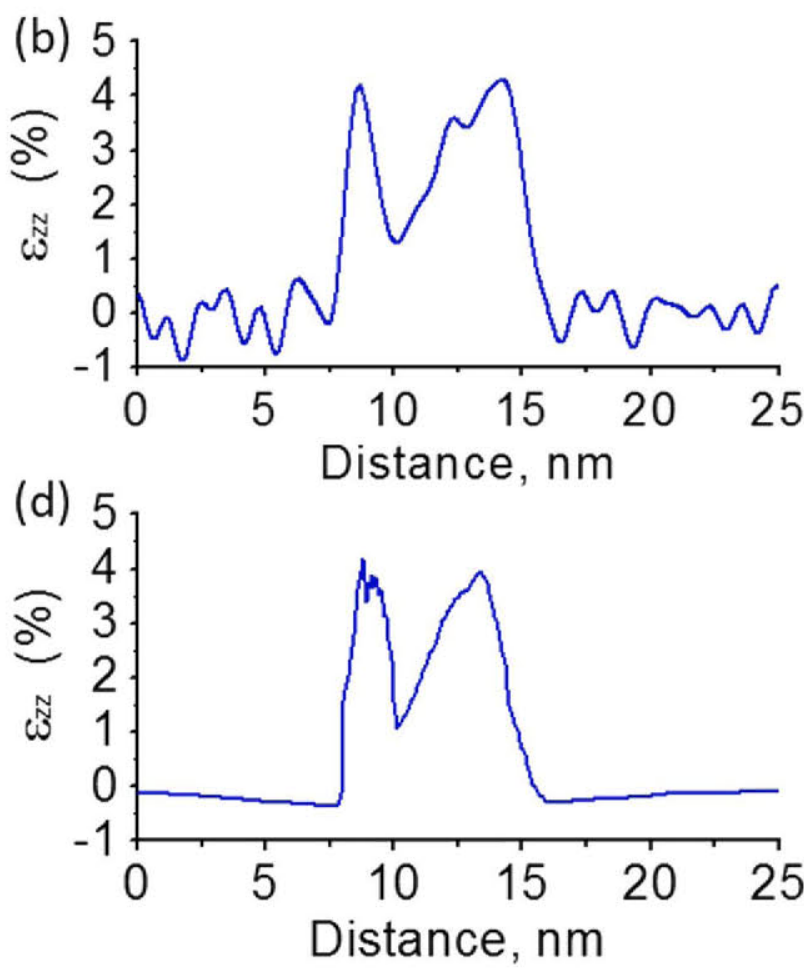

Figure 7. Deformation maps $\left(\varepsilon_{\mathrm{zz}}\right)$ of Sb28-RTA sample obtained from a HRTEM image acquired at the pole [110] (a) and from FEM simulations using structural and compositional data derived from TEM analysis (c), and their deformation profiles $\left(\varepsilon_{\mathrm{zz}}\right)(\mathrm{b})$ and (d), respectively. Color scale to $\varepsilon_{\mathrm{zz}}$ deformation expressed in percent.

between them. Nevertheless, although the difference between absolute contents is small, the volume of the region over the QDs in sample Sb28-RTA is about three times larger than in the sample Sb28, pointing to $\mathrm{Sb}$ accumulation.

Following this analysis, a model was constructed in which the Sb content was fixed to $29 \%$ in the area over the QD. Figure 7 shows the experimental and simulated deformation maps, $\varepsilon_{z z}$, after integrating over a TEM specimen thickness of $40 \mathrm{~nm}$ for Sb28-RTA. Profiles taken along the growth direction show a good agreement between experimental and simulated results. This final model is consistent with all experimental data and shows a similar $\mathrm{Sb}$ content over the QD as well as Ga incorporation into the QD as a result of In-Ga intermixing.

The reduction in the height of the QDs can only partially explain the blueshift so it is necessary to consider the change in composition of QDs produced by the In-Ga intermixing [36]. Our results from TEM and FEM analysis of the QDs suggest an increase of the Ga content inside the QDs of about $20 \%$ and an increase of the QD average volume of $30 \%$. This is consistent with previous electronic band structure calculations $(8 \times 8 \mathrm{kp}$ simulation) [19], which estimated that the content of Ga in the QDs must be increased by at least $27 \%$ to be consistent with the strong blueshift observed. In addition, although we have observed an enlargement of the Sb-rich region over the QDs, the $\mathrm{Sb}$ contents are similar to the asgrown sample. This mechanism leaves the type II alignment without major changes, with the ground state of the holes located outside the QDs near the QD-SRL interface. However, there is an improvement of the homogeneity of the $\mathrm{Sb}$ distribution in the SRL and the valence band discontinuity at the InAs-GaAsSb interface is smoothed by the $\mathrm{In} / \mathrm{Ga}$ alloy intermixing.

On the other hand, the principal problem associated with MBE-grown Sb-based III-V semiconductors is the high level of group III antisites $\left(\mathrm{As}_{\mathrm{Ga}}\right.$ ), which have been postulated to be the dominant structural defect in these materials [37, 38] though other non-radiative recombination centers linked to $\mathrm{Sb}_{\mathrm{Ga}}$ heteroantisite defects have also been reported [39]. The RTA induces a high reduction of the density of the defects $[40,41]$ as well as an improvement of the size and compositional uniformity of the QDs and SRL. All these facts explain the enhancement of the PL intensity and the narrowing of the QD PL emission peak in the annealed sample.

\section{Conclusions}

The study allowed us to determine the changes in the InAs QDs and GaAsSb SRL produced by RTA and to relate them to the observed optical properties. The annealing produces a considerable improvement of the PL spectrum but at the expense of an undesired blueshift. During annealing, Sb segregates away from the SRL resulting in a reduction in the SRL thickness, though conserving the maximum content (around 20\%) and improving its homogeneity. Part of this Sb aggregates to form $\mathrm{Sb}$-rich clusters around $15 \mathrm{~nm}$ in diameter that generate loop dislocations. In addition, the $\mathrm{Sb}$ is also accumulated near the QDs, making the layer adapt to the QD shape. FEM calculations allow us to estimate that the $\mathrm{Sb}$ 
content over the QD does not change a lot as a result of RTA, being close to the nominal contents and allowing the structure to preserve a similar type II band alignment after RTA. Simultaneously, a strong cation exchange (In and $\mathrm{Ga}$ ) between the QDs and the capping layer occurs. As a result, the QDs adopt a flatter shape with a smaller height and an increase in $\mathrm{Ga}$ content of about $20-30 \%$, which mainly explains the strong blueshift observed in the PL spectrum. Improvements in the quality of the GaAsSb layer around the QDs and on the homogeneity of the QD heights could explain the enhancement of the PL emission by the RTA.

\section{Acknowledgments}

We acknowledge the Spanish MICINN-MINECO for funding through project MAT2010-15206, MAT2013-47102-C2, and SCCYT-UCA for technical support.

\section{References}

[1] Zhang X Q, Ganapathy S, Kumano H, Uesugi K and Suemune I 2002 Photoluminescence study of InAs quantum dots embedded in GaNAs strain compensating layer grown by metalorganic-molecular-beam epitaxy J. Appl. Phys. 92 6813-8

[2] Liu H Y, Steer M J, Badcock T J, Mowbray D J, Skolnick M S, Navaretti P, Groom K M, Hopkinson M and Hogg R A 2005 Long-wavelength light emission and lasing from InAsGaAs quantum dots covered by a GaAsSb strain-reducing layer Appl. Phys. Lett. 86 1-3

[3] Yeh N T, Nee T E, Chyi J I, Hsu T M and Huang C C 2000 Matrix dependence of strain-induced wavelength shift in self-assembled InAs quantum-dot heterostructures Appl. Phys. Lett. 76 1567-9

[4] Shin H, Kim J B, Yoo Y H, Lee W, Yoon E and Yu Y M 2006 Enhanced strain of InAs quantum dots by an InGaAs ternary layer in a GaAs matrix J. Appl. Phys. 99 1-4

[5] Nabetani Y, Matsumoto T, Sasikala G and Suemune I 2005 Theory of strain states in InAs quantum dots and dependence on their capping layers $J$. Appl. Phys. 98 $063502-7$

[6] Ulloa J M, Drouzas I W D, Koenraad P M, Mowbray D J, Steer M J, Liu H Y and Hopkinson M 2007 Suppression of InAs/GaAs quantum dot decomposition by the incorporation of a GaAsSb capping layer Appl. Phys. Lett. 90 213103-5

[7] Ripalda J M, Granados D, González Y, Sánchez A M, Molina S I and García J M 2005 Room temperature emission at $1.6 \mu \mathrm{m}$ from InGaAs quantum dots capped with $\mathrm{GaAsSb}$ Appl. Phys. Lett. 87202108

[8] Marent A, Geller M, Schliwa A, Feise D, Pötschke K, Bimberg D, Akçay N and Öncan N 2007106 years extrapolated hole storage time in $\mathrm{GaSb} / \mathrm{AlAs}$ quantum dots Appl. Phys. Lett. 91242109

[9] Geller M, Kapteyn C, Müller-Kirsch L, Heitz R and Bimberg D 2003 Hole storage in $\mathrm{GaSb} / \mathrm{GaAs}$ quantum dots for memory devices Phys. Status Solidi B 238 258-61

[10] Laghumavarapu R B, Moscho A, Khoshakhlagh A, El-Emawy M, Lester L F and Huffaker D L 2007 $\mathrm{GaSb} / \mathrm{GaAs}$ type II quantum dot solar cells for enhanced infrared spectral response Appl. Phys. Lett. 90173125
[11] Tatebayashi J, Khoshakhlagh A, Huang S H, Balakrishnan G, Dawson L R, Huffaker D L, Bussian D A, Htoon H and Klimov V 2007 Lasing characteristics of GaSb/GaAs selfassembled quantum dots embedded in an InGaAs quantum well Appl. Phys. Lett. 90261115

[12] Montes Bajo M, Ulloa J M, del Moral M, Guzman A and Hierro A 2011 Near infrared InAs/GaAsSb quantum dot light emitting diodes Quantum Electronics, IEEE Journal of 47 1547-56

[13] Chow W W and Schneider H C 2001 Charge-separation effects in $1.3 \mu \mathrm{m}$ GaAsSb type-II quantum-well laser gain Appl. Phys. Lett. 78 4100-2

[14] Utrilla A, Ulloa J, Guzman A and Hierro A 2013 Impact of the $\mathrm{Sb}$ content on the performance of GaAsSb-capped InAs/ GaAs quantum dot lasers Appl. Phys. Lett. 103111114

[15] Teissier R, Sicault D, Harmand J C, Ungaro G, Le Roux G and Largeau L 2001 Temperature-dependent valence band offset and band-gap energies of pseudomorphic GaAsSb on GaAs J. Appl. Phys. 89 5473-7

[16] Ulloa J M et al 2007 Atomic scale study of the impact of the strain and composition of the capping layer on the formation of InAs quantum dots J. Appl. Phys. 101 081706-7

[17] Haxha V, Drouzas I, Ulloa J M, Bozkurt M, Koenraad P M, Mowbray D J, Liu H Y, Steer M J, Hopkinson M and Migliorato M A 2009 Role of segregation in InAs/GaAs quantum dot structures capped with a GaAsSb strainreduction layer Phys. Rev. B-Cond. Mat. Mater. Phys. 80 165334

[18] Liao Y A, Hsu W T, Chiu P C, Chyi J I and Chang W H 2009 Effects of thermal annealing on the emission properties of type-II InAs/GaAsSb quantum dots Appl. Phys. Lett. 94 053101

[19] Ulloa J M, Llorens J M, Alen B, Reyes D F, Sales D L, Gonzalez D and Hierro A 2012 High efficient luminescence in type-II GaAsSb-capped InAs quantum dots upon annealing Appl. Phys. Lett. 101 253112-5

[20] Williamson A J, Wang L W and Zunger A 2000 Theoretical interpretation of the experimental electronic structure of lens-shaped self-assembled InAs/GaAs quantum dots Phys. Rev. B 62 12963-77

[21] Yamauchi T, Matsuba Y, Bolotov L, Tabuchi M and Nakamura A 2000 Correlation between the gap energy and size of single InAs quantum dots on $\mathrm{GaAs}(001)$ studied by scanning tunneling spectroscopy Appl. Phys. Lett. 77 4368-70

[22] Ngo C Y, Yoon S F, Fan W J and Chua S J 2006 Effects of size and shape on electronic states of quantum dots Num Sim Semicond Optoelectron Devices, 2006 NUSOD '06 Int Conf; Sept. 2006 pp 51-2

[23] Chaldyshev V V, Kolesnikova A L, Bert N A and Romanov A E 2005 Investigation of dislocation loops associated with As-Sb nanoclusters in GaAs J. Appl. Phys. $97024309-10$

[24] Dominguez L, Reyes D F, Bastiman F, Sales D L, Richards R D, Mendes D, David J P R and Gonzalez D 2013 Formation of tetragonal InBi clusters in $\operatorname{InAsBi} / \operatorname{InAs}(100)$ heterostructures grown by molecular beam epitaxy Appl. Phys. Express 6112601

[25] Beanland R 2005 Dark field transmission electron microscope images of III-V quantum dot structures Ultramicroscopy 102 $115-25$

[26] Wahra A, Tan C H, Xie J, Vines P and Beanland R 2011 Three-dimensional measurement of composition changes in InAs/GaAs quantum dots J. Phys.: Conf. Series 326

[27] Sanchez A M, Beltran A M, Beanland R, Ben T, Gass M H, De La Pẽa F, Walls M, Taboada A G, Ripalda J M and Molina S I 2010 Blocking of indium incorporation by antimony in III-V-Sb nanostructures Nanotech. 21145606 
[28] Hernández-Maldonado D et al 2011 Compositional analysis with atomic column spatial resolution by 5 th-order aberration-corrected scanning transmission electron microscopy Microsc. Microanal. 17 578-81

[29] Reyes D, Gonzalez D, Ulloa J, Sales D, Dominguez L, Mayoral A and Hierro A 2012 Impact of N on the atomicscale $\mathrm{Sb}$ distribution in quaternary GaAsSbN-capped InAs quantum dots Nano Res. Lett. 7653

[30] Herrera M et al 2009 Atomic scale high-angle annular dark field STEM analysis of the $\mathrm{N}$ configuration in dilute nitrides of GaAs Phys. Rev. B 80125211

[31] Grillo V, Carlino E and Glas F 2008 Influence of the static atomic displacement on atomic resolution Z-contrast imaging Phys. Rev. B 77054103

[32] Hÿtch M J, Snoeck E and Kilaas R 1998 Quantitative measurement of displacement and strain fields from HREM micrographs Ultramicroscopy 74 131-46

[33] Bozkurt M, Ulloa J and Koenraad P 2011 An atomic scale study on the effect of Sb during capping of MBE grown IIIV semiconductor QDs Semicond Sci. Tech. 26064007

[34] Biasiol G and Heun S 2011 Compositional mapping of semiconductor quantum dots and rings Phys. Rep. $\mathbf{5 0 0}$ $117-73$

[35] Lemaitre A, Patriarche G and Glas F 2004 Composition profiling of InAs/GaAs quantum dots Appl. Phys. Lett. 85 3717-9
[36] Ilahi B, Sfaxi L and Maaref H 2007 Optical investigation of InGaAs-capped InAs quantum dots: Impact of the straindriven phase separation and dependence upon post-growth thermal treatment J. Lumin. 127 741-6

[37] Brewer P D, Chow D H and Miles R H 1996 Atomic antimony for molecular beam epitaxy of high quality III-V semiconductor alloys J. Vacuum Sci. Tech. B 14 2335-8

[38] Wicaksono S, Yoon S F, Loke W K, Tan K H, Lew K L, Zegaoui M, Vilcot J P, Decoster D and Chazelas J 2007 Effect of growth temperature on defect states of $\mathrm{GaAsSbN}$ intrinsic layer in $\mathrm{GaAs} / \mathrm{GaAsSbN} / \mathrm{GaAs}$ photodiode for $1.3 \mu \mathrm{m}$ application J. Appl. Phys. 102044505

[39] Cheah W K, Fan W J, Yoon S F, Tan K H, Liu R and Wee A T S 2005 Surfactant and impurity properties of antimony on GaAs and $\mathrm{GaAs}_{1-\mathrm{x}} \mathrm{N}_{\mathrm{x}}$ on GaAs [100] by solid source molecular beam epitaxy Thin Solid Films $\mathbf{4 8 8}$ $56-61$

[40] Dang Y X, Fan W J, Ng S T, Wicaksono S, Yoon S F and Zhang D H 2005 Study of interdiffusion in GaAsSbN/GaAs quantum well structure by ten-band k.p method $J$. Appl. Phys. 98026102

[41] Zhao H, Xu Y Q, Ni H Q, Zhang S Y, Wu D H, Han Q, Wu R H and Niu Z C 2006 Characteristic of rapid thermal annealing on $\mathrm{GaIn}(\mathrm{N})(\mathrm{Sb}) \mathrm{As} / \mathrm{GaAs}$ quantum well grown by molecular-beam epitaxy J. Appl. Phys. 99034903 